\title{
Introductory notes for the Acta IMEKO fourth issue 2021
}

\author{
Francesco Lamonaca ${ }^{1}$ \\ ${ }^{1}$ University of Calabria, Dept. of Computer Science, Modelling, Electronic and System, via P.Bucci 41C, Arcavacata di Rende, 87036 (CS), \\ Italy
}

Section: EDITORIAL

Citation: Francesco Lamonaca, Introductory notes for the Acta IMEKO fourth issue 2021, Acta IMEKO, vol. 10, no. 4, article 1, December 2021, identifier: IMEKO-ACTA-10 (2021)-04-01

Received December 23, 2021; In final form December 23, 2021; Published December 2021

Copyright: This is an open-access article distributed under the terms of the Creative Commons Attribution 3.0 License, which permits unrestricted use, distribution, and reproduction in any medium, provided the original author and source are credited.

Corresponding author: Francesco Lamonaca, e-mail: editorinchief.actaimeko@hunmeko.org

\section{Dear Readers,}

Also in this issue, high quality and heterogeneous papers are presented confirming Acta IMEKO as the natural platform for disseminating measurement information and stimulating collaboration among researchers from many different fields. I hope you will enjoy your reading.

As usual, the General Track collects contributions that do not relate to a specific event. As Editor in Chief, it is my pleasure to give an overview of these papers, with the aim of encouraging potential authors to consider sharing their research through Acta IMEKO.

An interesting technique is proposed by Oleksandr Vasilevskyi et al. in the paper 'Indicators of reproducibility and suitability for assessing the quality of production services' for estimating the probability of the possible appearance of defective products or the inconsistency of the production service on the basis of indexes of suitability and reproducibility of the production process. The accuracy of the proposed estimation methodology, which includes the proposed mathematical apparatus, was estimated on the basis of the correctness index, whose assessment method is carried out in accordance with the international standard ISO 57252:2002.

Mohammed Alktranee et al. in the paper 'Simulation study of the photovoltaic panel under different operation conditions' study the effects of temperature distribution on the photovoltaic panel at different solar radiation values and temperatures under various operative conditions in January and July. A 3D model of the PV panel was simulated using ANSYS software, depending on the various values of temperature and solar radiation values obtained using mathematic equations.

In cognitive radio systems, estimating primary user direction of arrival (DOA) measurement is one of the key issues. In order to increase the probability detection, multiple sensor antennas are used and they are analysed by using subspace-based technique. In the paper 'An adaptive learning algorithm for spectrum sensing based on direction of arrival estimation in cognitive radio systems', Sala Surekha et al. considered wideband spectrum with sub channels and here each sub channel facilitated with a sensor for the estimation of DOA. In practical spectrum sensing process interference component also encounters in the sensing process. To avoid this interference level at output of receiver, the authors used an adaptive learning algorithm known as Normalized Least Absolute Mean Deviation (NLAMD) algorithm.

Rosario Morello et al. in the paper 'Design of a non-invasive sensing system for diagnosing gastric disorders' present a smart EGG (Electrogastrography) sensing system to non-invasively diagnose gastric disorders. In detail, the system records the gastric slow waves by means of skin surface electrodes placed in the epigastric area. Cutaneous myoelectrical signals are so acquired from the body surface in proximity of stomach. Electro-gastrographic record is then processed. According to the diagnostic model designed from the authors, the system estimates specific diagnostic parameters in time and frequency domains. It uses Discrete Wavelet Transform to obtain power spectral density diagrams. The frequency and power of the EGG waveform and the dominant frequency components are so analysed. The defined diagnostic parameters are compared to the reference values of a normal EGG in order to estimate the presence of gastric pathologies by the analysis of arrhythmias (tachygastria, bradygastria and irregular rhythm).

For military divers, having a robust, secure, and undetectable wireless communication system available is a fundamental need. Wireless intercoms using acoustic waves are currently used. These systems, even if reliable, have the limit of being easily identifiable and detectable. Visible light can pass through sea water. Therefore, light can be used to develop short-range wireless communication systems. To realize secure close-range underwater wireless communication, the Underwater Optical Wireless Communication (UOWC) can be a valid alternative to acoustic wireless communication. UOWC is not a new idea, but the problem of the presence of sunlight and the possibility of using near-ultraviolet radiation (near-UV) has not been adequately addressed in the literature yet. In military applications, the possibility of using invisible optical radiation can be of great interest. In the paper ' $L E D$-to-LED wireless communication between divers' by Fabio Leccese et al., a feasibility study is carried out to 
demonstrate that UOWC can be performed using nearultraviolet radiation. The proposed system can be useful for wireless voice communications between military divers as well as amateur divers

Moreover, this issue contains extended version of selected papers presented to one of the most important International and Italian metrology events organized in Italy: the 2020 IMEKO TC19 International Workshop on Metrology for the Sea "Learning to measure sea health parameters", guest edited by Prof. Silvio del Pizzo; the International Excellence Italo Gorini Ph.D School guest edited by Prof. Pasquale Arpaia and Dr. Umberto Cesaro; the 40th Measurement Day jointly organised by the Italian associations Italian group of electric and electronic measurements (GMEE) and Italian group of Mechanical and
Thermal Measurements (GMTT) guest edited by Prof. Carlo Carobbi, Prof. Nicola Giaquinto, Prof. Gian Marco Revel. The XXIX Italian National Congress on Mechanical and Thermal Measurements guest edited by Prof. Alfredo Cigada and Prof. Roberto Montanini.

The strong Italian contribution to this fourth issue is justified by the wish of the Italian Metrology Community to support IMEKO and its journals.

I hope that in the near future Acta IMEKO will receive an equally important contribution from the other IMEKO member Countries and beyond.

Francesco Lamonaca

Editor in Chief 